\title{
Análisis Histopatológico del Cartílago Articular Expuesto a Metilprednisolona, Ropivacaína y Ácido Hialurónico en un Modelo Animal de Osteoartrosis
}

\author{
Histopathological Analysis of the Articular Cartilage Exposed to Methylprednisolone, \\ Ropivacaine and Hyaluronic Acid in an Animal Model of Osteoarthrosis \\ Ranmel Bustos Latabán*; Iván Alberto Marino Martínez**; Víktor Javier Romero Díaz ***; \\ Oscar Fernando Mendoza Lemus*; Jorge Lara Arias* \& Santiago De La Garza Castro*
}

BUSTOS, L. R.; MARINO, M. I. A.; ROMERO, D. V. J.; MENDOZA, L. O. F.; LARA, A. J. \& DE LA GARZA, C. S. Análisis histopatológico del cartílago articular expuesto a metilprednisolona, ropivacaína y ácido hialurónico en un modelo animal de osteoartrosis. Int. J. Morphol., 32(4):1199-1206, 2014.

RESUMEN: La osteoartrosis es un padecimiento del aparato locomotor con una prevalencia elevada y en crecimiento, paralela al envejecimiento de la población. La infiltración intraarticular de sustancias para aliviar la sintomatología de la osteoartrosis es una práctica común en el consultorio médico de los especialistas que atienden esta enfermedad. Aunque la sintomatología mejora con la infiltración de anestésicos locales, corticoesteroides y suplementos viscosantes, es aún incierto el efecto que estas sustancias tienen sobre la integridad del cartílago articular. Este estudio explora a nivel macroscópico e histológico el efecto de la infiltración de ropivacaína, metilprednisolona y ácido hialurónico sobre el cartílago articular en un modelo de osteoartrosis química en conejos (n=24). Nuestros resultados indican que en los grupos infiltrados con metilprednisolona $(n=8)$ y ropivacaína $(n=8)$ la estructura del cartílago articular presento alteraciones más severas con respecto a su grupo control, además de una disminución importante en la síntesis de matriz extracelular. En el grupo infiltrado con ácido hialurónico $(n=8)$, las alteraciones macroscópicas e histológicas del cartílago articular mejoraron con respecto a su grupo control, presentando una estructura integra y síntesis de matriz extracelular normal.

PALABRAS CLAVE: Infiltración intraarticular; Cartílago articular; Osteoartrosis.

\section{INTRODUCCIÓN}

La osteoartrosis es una de las enfermedades más prevalentes del aparato locomotor, y constituye una de las causas más frecuentes de incapacidad, especialmente en los adultos mayores. Se calcula que afecta al $14 \%$ de la población y que más del $80 \%$ de los mayores de 65 años presentan signos radiográficos de artrosis, al menos, en una articulación. Felson et al. (2000) Su impacto socioeconómico se incrementará en las próximas décadas de manera paralela al envejecimiento de la población (Elders, 2000).

La osteoartrosis no es una enfermedad única, sino que constituye un grupo heterogéneo de enfermedades con características comunes, secundarias a alteraciones bioquímicas y biomecánicas que afectan al metabolismo del cartílago (Brandt et al., 1998). La osteoartrosis se clasifica en primaria o secundaria según la presencia o no, de una causa o factor predisponente. Clínicamente, se manifiesta por dolor, pérdida de movilidad, limitación funcional, crepitación, derrames ocasionales e inflamación sin alteraciones sistémicas, aunque no siempre existe correlación entre la clínica y los hallazgos radiológicos (Kuettner \& Goldberg, 1995).

En la etapa inicial de la osteoartrosis, la superficie del cartílago articular se vuelve irregular y con pequeñas hendiduras en las capas superficiales. Conforme progresa la enfermedad, las irregularidades se hacen más grandes y aparecen "fisuras verticales" que se extienden a las capas más profundas, el cartílago articular se erosiona y en los estadios más avanzados, deja expuesto el hueso subcondral (Meachim, 1972). La histología muestra a los condrocitos en apoptosis adyacentes a las zonas de fibrilación y hendi-

* Hospital Universitario, Servicio de Ortopedia y Traumatología, Universidad Autónoma de Nuevo León, Nuevo León, México..

** Facultad de Medicina, Departamento de Patología, Universidad Autónoma de Nuevo León, Nuevo León, México.

**** Facultad de Medicina, Departamento de Histología, Universidad Autónoma de Nuevo León, Nuevo León, México. 
BUSTOS, L. R.; MARINO, M. I. A.; ROMERO, D. V. J.; MENDOZA, L. O. F.; LARA, A. J. \& DE LA GARZA, C. S. Análisis histopatológico del cartílago articular expuesto a metilprednisolona, ropivacaína y ácido hialurónico en un modelo animal de osteoartrosis. Int. J. Morphol., 32(4):1199-1206, 2014.

duras con una evidente disminución de la celularidad. En un intento de reparación, también coexisten otras zonas de proliferación condrocitaria, con formación de clones o racimos e hipertrofia celular (Pelletier et al., 2000; Poole et al., 1991). La matriz extracelular presenta alteraciones en la producción y degradación de la misma. En las zonas lesionadas, proliferan fibrocitos que inducen metaplasia del cartílago hialino hacia fibrocartílago. La membrana sinovial presenta una reacción inflamatoria, especialmente en las fases avanzadas de la enfermedad (Saito et al., 2002). La cápsula y los ligamentos desarrollan también una reacción inflamatoria que puede evolucionar hacia una excesiva laxitud o fibrosis con mayor frecuencia, ambos fenómenos contribuyen a distorsionar la biomecánica articular. Los meniscos presentan lesiones en los que, como en otros tejidos articulares, se evidencian intentos reparativos, con proliferación e hipertrofia de fibroblastos (Fahmy et al.).

La infiltración articular representa una práctica útil que puede llevarse a cabo por médicos de ortopedia y traumatología y por otros médicos entrenados. Estas técnicas ayudan a aliviar el dolor y mejoran la funcionalidad del paciente, al mismo tiempo que amplían las opciones terapéuticas del médico y complementan otros tratamientos. Existen numerosas indicaciones para realizar una infiltración articular, durante el diagnóstico por ejemplo para realizar una exploración sin dolor y durante el tratamiento con diferentes objetivos como aliviar la sintomatología, detener la progresión de la enfermedad, etc.

Entre las sustancias que pueden ser infiltradas en la articulación se encuentran los anestésicos locales, los corticoesteroides y suplementos viscosantes como el Ácido Hialurónico; así pues es importante conocer los efectos clínicos y biológicos que producen estas sustancias al ser infiltrados en la articulación.

El objetivo de este estudio fue observar el efecto histopatológico de la infiltración intraarticular con Metilprednisolona, Ropivacaína y Ácido Hialurónico sobre el cartílago articular en un modelo animal de artrosis química.

\section{MATERIAL Y MÉTODO}

El presente estudio contó con la aprobación del Comité de ética y Comité de investigación de la Facultad de Medicina y Hospital Universitario de la Universidad Autónoma de Nuevo León con número de registro OR12-001.

Modelo animal de osteoartrosis química. Se emplearon 24 conejos, machos sanos, de 3 meses de edad y 2,5 kg de peso promedio. A cada conejo se le aplicó una inyección intramuscular de Xilacina 1,9 mg/Kg y Ketamina 46 mg/ $\mathrm{Kg}$. Posterior a la sedación, se trataron ambas rodillas con una infiltración de $250 \mu \mathrm{l}$ de colagenasa tipo II (Clostridium histolyticum tipo II, enzima activa $425 \mathrm{U} / \mathrm{mg}$, SIGMA ALDRICH) a una concentración de $4 \mathrm{mg} / \mathrm{ml}$ para establecer una osteoartrosis leve de acuerdo al método estandarizado por Kikuchi et al. (1998). El día de la primera infiltración con colagenasa se estableció como día 1 y una segunda infiltración fue realizada al día 4. Los animales de experimentación se mantuvieron durante 3 semanas en bioterio para establecer la osteoartrosis leve (Kikuchi et al.).

Infiltración de sustancias en estudio. Posterior al establecimiento del modelo de osteoartrosis leve, las rodillas derechas de cada conejo se infiltraron con una de las sustancias de estudio. Un primer grupo de 8 conejos recibieron en su rodilla derecha una dosis única de $0,6 \mathrm{ml}$ de Ácido Hialurónico (Hilano G-F 20, Hilano A e Hilano B) a una concentración de Hilano A 8,0 mg/ml e Hilano B 2,0 mg/ $\mathrm{ml}$. El segundo grupo de 8 conejos recibieron en su rodilla derecha una dosis única de $0,6 \mathrm{ml}$ de Acetato de Metilprednisolona (Depo-Medrol®) a una concentración 40 $\mathrm{mg} / \mathrm{ml}$, y el tercer grupo de 8 conejos recibieron en su rodilla derecha una dosis única de $0,6 \mathrm{ml}$ de Ropivacaína (Naropin ${ }^{\circledR}$ ) a una concentración 7,5 mg/ml. Todas las rodillas izquierdas recibieron $0,6 \mathrm{ml}$ de solución fisiológica $(\mathrm{NaCl} 0,9 \%)$ y fueron empleadas como grupo control sin tratamiento.

Todos los procedimientos fueron realizados bajo anestesia general con Xilacina y Ketamina. Todas las rodillas fueron infiltradas por el mismo investigador. Los conejos se mantuvieron en bioterio bajo los mismos cuidados higiénicos y dietéticos durante 4 semanas.

Una vez concluido el periodo de exposición a las sustancias en estudio, se realizó el sacrificio de los animales de experimentación mediante una sobredosis de Xilacina y Ketamina intravenosa.

Estudios histológicos. Inmediatamente después del sacrificio, se realizó la disección y resección en bloque de ambas rodillas de cada uno de los 24 conejos del estudio. Cada rodilla se sumergió en una solución de formol al 10\% para su preservación. Las muestras de ambas rodillas fueron decalcificadas y procesadas mediante la técnica de rutina para inclusión en parafina. Se realizaron cortes sagitales de 4 micrómetros y se tiñeron con Hematoxilina y Eosina para la valoración de estructura del cartílago, la celularidad e integridad del "tidemark". También se empleó la tinción con Safranina-O y Verde rápido para la valoración de los componentes de la matriz extracelular. 
Análisis morfológico macroscópico. Después de la disección completa de las rodillas se realizó el análisis morfológico empleando como guía la escala publicada por Yoshioka et al. (1996), en la cual el grado 1 indica superficie articular intacta; grado 2, mínima fibrilación; grado 3, fibrilación evidente y grado 4, erosión con exposición de hueso (Tabla I).

Tabla I. Escala de Yoshioka para la evaluación macroscópica del cartílago articular.

\begin{tabular}{ll}
\hline Grado & Descripción \\
\hline $\mathbf{1}$ & Superficie articular intac ta \\
$\mathbf{2}$ & Mínima fibrilación \\
$\mathbf{3}$ & Fibrilación evidente \\
$\mathbf{4}$ & Erosión con exposición de hueso \\
\hline
\end{tabular}

El análisis morfológico macroscópico fue realizado para ambas rodillas de los 24 conejos por un mismo evaluador en un diseño de ciego con respecto al evaluador.

Análisis histopatológico. La valoración histopatológica de la lesión generada por la colagenasa II en el cartílago y la posible protección de las sustancias en estudio, se realizó mediante la escala propuesta por Mankin et al. (1971) (Piper et al., 2008). La escala de Mankin asigna una puntuación histológica a cada caso como resultado de sumar los valores correspondientes a las alteraciones en a) la estructura del cartílago, b) la celularidad, c) la tinción de la matriz y d) la integridad del "tidemark" o línea limitante basófila. De este modo, al cartílago normal le corresponden 0 puntos, y a la afectación más grave del cartílago le correspon-

Tabla II. Escala de Mankin para la evaluación histopatológica del cartílago articular.

\begin{tabular}{llc}
\hline Subesc alas & Alteraciones & Puntuación \\
\hline & Normal & 0 \\
& Irregularidades en la superficie & 1 \\
& Irregularidades en la superficie y pannus & 2 \\
Estructura & Hendiduras hasta zona de transición & 3 \\
& Hendiduras hasta zona radial & 4 \\
& Hendiduras hasta zona calcificada & 5 \\
& Desorganiza ción completa & 6 \\
& Normal & 0 \\
Celularidad & Hipercelularidad difusa & 1 \\
& Presencia de clones & 2 \\
& Hipocelularidad & 3 \\
& Normal & 0 \\
Tinción de & Reducción leve & 1 \\
la matriz & Reducción moderada & 2 \\
& Reducción severa & 3 \\
Integridad & Ausencia de tinción & 4 \\
"tidemark" & Invasta & 0 \\
& & 1
\end{tabular}

derían 14 puntos (Tabla II). La evaluación histopatológica fue realizada por un observador independiente que se mantuvo sin conocer el grupo al que pertenecía cada muestra. La escala de Mankin ha demostrado buena correlación inter e intra observador, refleja adecuadamente el nivel de alteración metabólica condrocitaria y constituye el Gold standard para la evaluación histopatológica del cartílago hialino articular.

Análisis estadístico. El cálculo del tamaño de muestra para el modelo animal, alfa de 0,05 , beta 0,08 , una dirección, fue de 6 individuos por grupo (Kikuchi et al.). El cálculo de tamaño de muestra para comparación de medias relacionadas, alfa de 0,05 , beta 0,08 , dos direcciones, tomando una diferencia mínima de 2.5 , fue de 7 individuos por grupo. Se realizó estadística descriptiva con el cálculo de la media, mediana, desviación estándar y rango de los datos.

Las comparaciones de los resultados de rodilla izquierda (control) contra rodilla derecha del mismo individuo y por grupo se realizaron por medio de prueba de Wilcoxon para grupos relacionados. La significancia alfa se determinó en 0.05 .

El procesamiento electrónico de los datos y métodos de estadística descriptiva e inferencial, se llevó cabo con el programa STATA-10-08, licencia intermed-08.

\section{RESULTADOS}

Análisis morfológico macroscópico. Los resultados del análisis morfológico macroscópico empleando la escala de Yoshioka se resumen en la Tabla III.

En el grupo que se infiltro con Ácido Hialurónico se observó que las rodillas derechas infiltradas presentaron un cartílago articular intacto o con mínima fibrilación mientras que su grupo control (rodillas izquierdas) presentó áreas extensas de cartílago articular con fibrilación evidente, con una diferencia estadísticamente significativa $(\mathrm{P}=0,025)$ (Figs. 1A y 1E). La evaluación macroscópica no reveló diferencia estadísticamente significativa entre la integridad del cartílago articular de las rodillas derechas infiltradas con Acetato de Metilprednisolona o Ropivacaína y sus respectivos grupos de control (Figs. 1B, 1C, 1E y 1F).

Análisis histopatológico. Tanto en los grupos tratados con Metilprednisolona como con Ropivacaína, la puntuación en la escala de Mankin de los cortes 


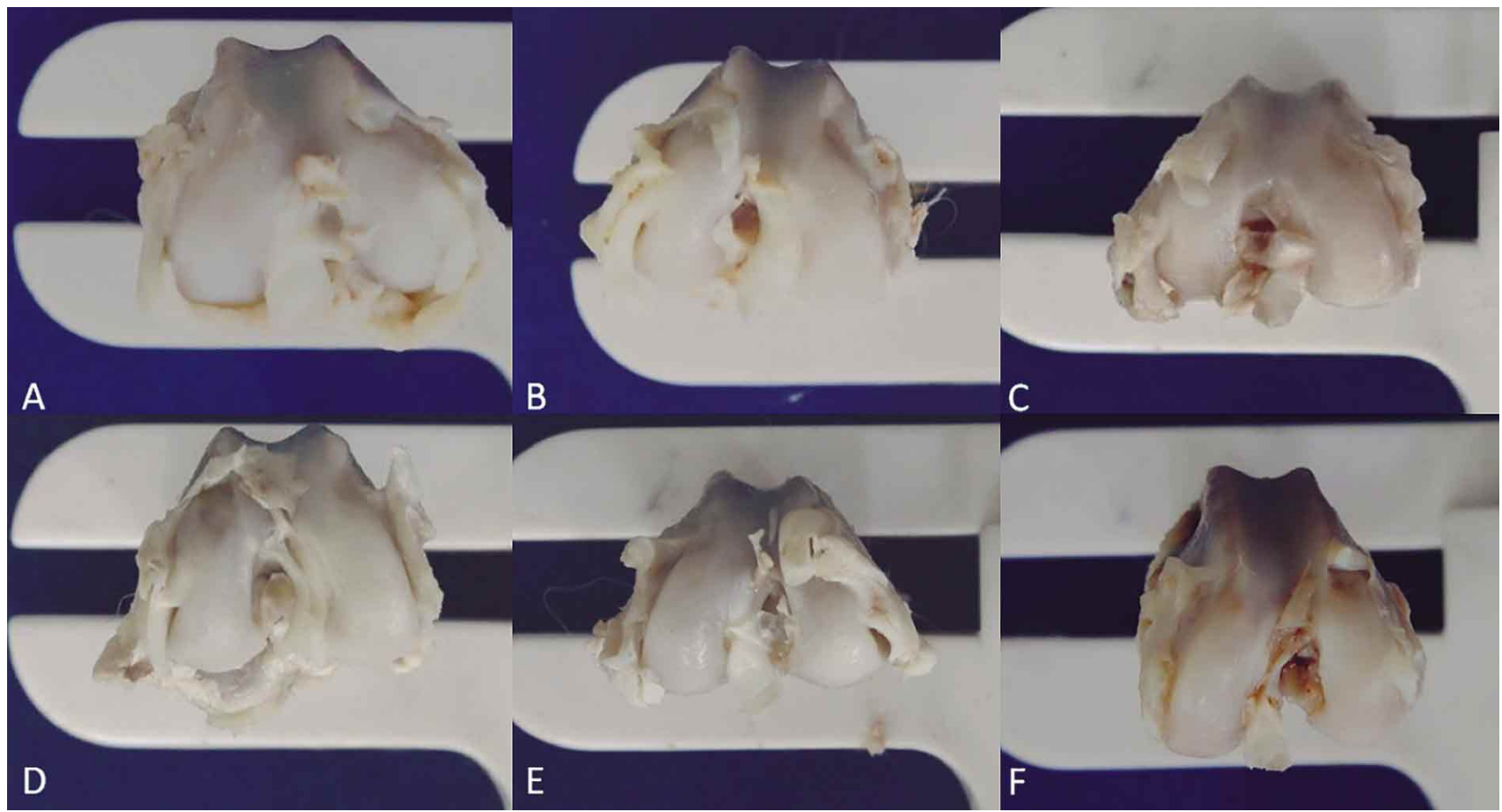

Figura 1. Análisis morfológico macroscópico. Rodillas derechas tratadas con ácido hialurónico (A), Metilprednisolona (B), Ropivacaína (C) y respectivas rodillas izquierdas control con Colagenasa (D, E y F).

histológicos de rodillas derechas fue mayor que el de sus respectivos controles, con una significancia estadística de $\mathrm{P}=0,010$ y $\mathrm{P}=0,008$, respectivamente. Lo anterior sugiere un daño adicional al daño causado por la Colagenasa tipo II al cartílago articular por el uso de estos dos agentes (Tabla IV).

En el grupo infiltrado con Metilprednisolona la principal diferencia histológica entre las rodillas derechas e izquierdas, fue en la afección a la estructura del cartílago, presentándose hendiduras de mayor profundidad en rodillas de- rechas $(\mathrm{P}=0,011)$ (Fig. 2B y 2D). Un hallazgo importante que se presentó en la mayoría de los cortes histológicos de cartílago articular de las rodillas derechas de este grupo fue la ausencia o importante disminución de tinción de la matriz extracelular con Safranina-O/Verde rápido, indicando la ausencia de proteoglicanos del cartílago (Fig. 2F).

Para el grupo infiltrado con Ropivacaína al igual que el grupo infiltrado con Metilprednisolona, las principales diferencias consistieron una mayor afección de la estructura del cartílago articular de las rodillas derechas, presen-

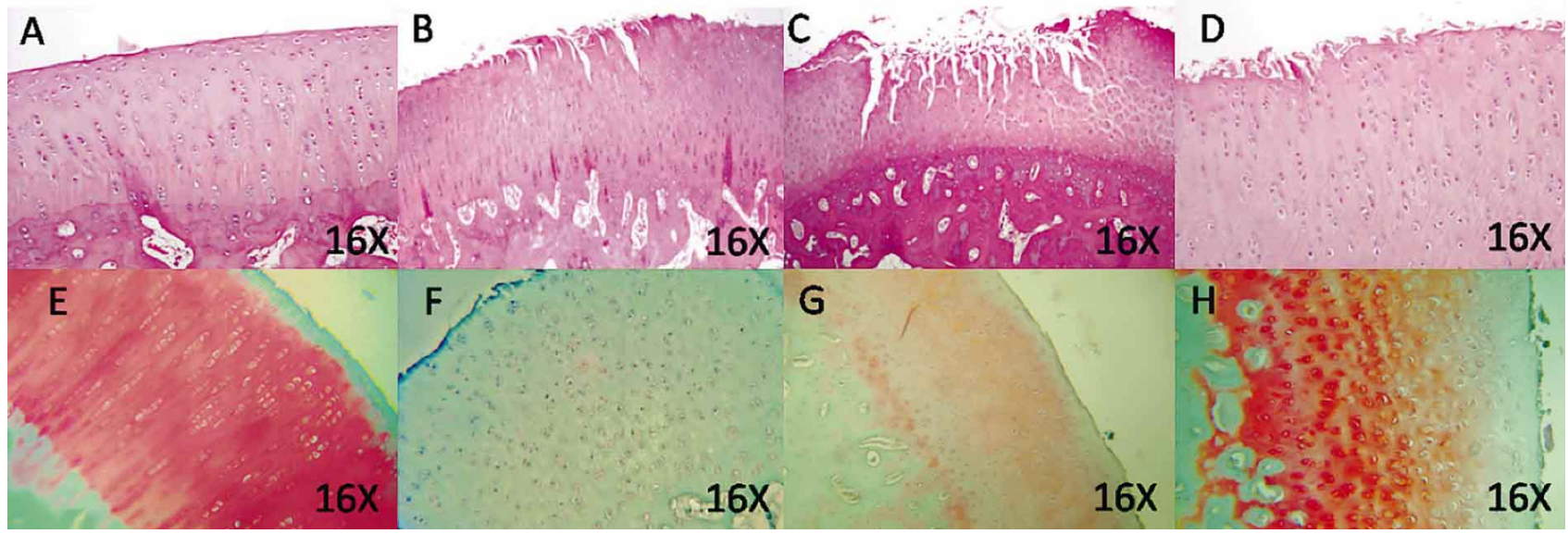

Fig. 2. Análisis histológico. Tinción con Hematoxilina/Eosina (A, B, C y D) y Safranina-O/Verde rápido (E, F, G y H) del cartílago articular de los grupos infiltrados con ácido hialurónico (A y E), Metilprednisolona (B y F), Ropivacaína (C y G) y control con Colagenasa (D y H). 
BUSTOS, L. R.; MARINO, M. I. A.; ROMERO, D. V. J.; MENDOZA, L. O. F.; LARA, A. J. \& DE LA GARZA, C. S. Análisis histopatológico del cartílago articular expuesto a metilprednisolona, ropivacaína y ácido hialurónico en un modelo animal de osteoartrosis. Int. J. Morphol., 32(4):1199-1206, 2014.

Tabla III. Resultados del análisis Morfológico Macroscópico (Escala de Yoshioka).

\begin{tabular}{llccccc}
\hline Grupo & & Media & DE & Mediana & Rango & Valor de p \\
\hline Metilprednisolona & Rodilla derecha & 2,00 & 0,76 & 2,00 & 2,00 & $>0,05$ \\
& Rodilla izquierda & 1,75 & 0,71 & 2,00 & 2,00 & \\
Ropivacaína & Rodilla derecha & 2,13 & 0,35 & 2,00 & 1,00 & $>0,05$ \\
& Rodilla izquierda & 1,75 & 0,46 & 2,00 & 1,00 & \\
Ácido Hialurónico & Rodilla derecha & 1,29 & 0,49 & 1,00 & 1,00 & 0,025 \\
& Rodilla izquierda & 2,00 & 0,00 & 2,00 & 0,00 & \\
\hline
\end{tabular}

Tabla IV. Resultados del Análisis histopatológico (Escala de Mankin).

\begin{tabular}{lllcccc}
\hline Grupo & & Media & DE & Mediana & Rango & Valor de p \\
\hline Metilprednisolona & Rodilla derecha & 8,25 & 1,04 & 8,00 & 3,00 & 0,010 \\
& Rodilla izquierda & 5,13 & 0,99 & 5,00 & 3,00 & \\
Ropivacaína & Rodilla derecha & 8,63 & 2,50 & 8,00 & 6,00 & 0,008 \\
& Rodilla izquierda & 5,38 & 1,06 & 5,50 & 3,00 & \\
Ácido Hialurónico & Rodilla derecha & 2,14 & 1,07 & 2,00 & 3,00 & 0,018 \\
& Rodilla izquierda & 6,71 & 1,25 & 7,00 & 4,00 & \\
\hline
\end{tabular}

tándose irregularidades en la superficie y hendiduras más profundas respecto a su grupo control $(\mathrm{P}=0,026)$ (Figs. $2 \mathrm{C}$ y 2D). La tinción de la matriz del cartílago con safranina-o/ verde rápido revelo una disminución de la presencia de proteoglicanos con respecto al control (Figs. $2 \mathrm{G}$ y $2 \mathrm{H}$ ). Exclusivamente en este grupo se observaron alteraciones en la celularidad en la mayoría de los casos e hipercelularidad $(\mathrm{P}=0,047)$ (Figs. 3A y 3C). Además de las alteraciones en la celularidad, se observó hiperplasia de la membrana sinovial, esta diferencia fue estadísticamente significativa al compararla con su grupo control en donde la sinovial se muestra normal $(\mathrm{P}=0,014)$ (Figs. 3B y 3D).
A diferencia de los grupos infiltrados con Metilprednisolona y Ropivacaína, el grupo infiltrado con Ácido Hialurónico presentó una menor puntuación en la escala de Mankin con respecto a su control, siendo esta diferencia estadísticamente significativa ( $\mathrm{P}=0,018)$ (Tabla IV). En este grupo se observan las estructuras del cartílago articular más intactas, con superficies más regulares, celularidad normal e integridad del "tidemark" normal (Fig. 2A). Además la tinción con Safranina-O/Verde rápido demostró una mayor presencia de proteoglicanos en la matriz extracelular del cartílago articular de las rodillas derechas con respecto a su control $(\mathrm{P}=0,016)$ (Figs. $2 \mathrm{E}$ y $2 \mathrm{H})$.

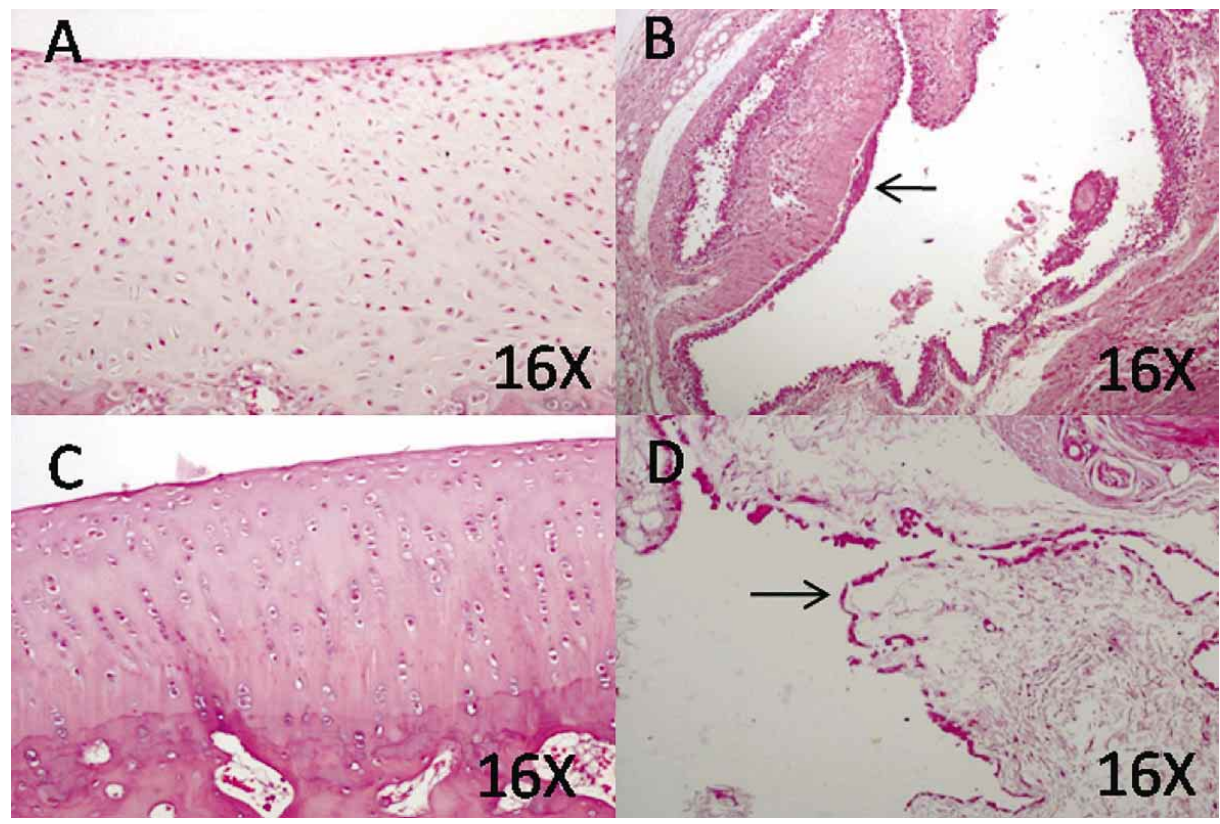

Fig. 3. Alteraciones únicas en grupo infiltrado con Ropivacaína. Tinción con Hematoxilina/Eosina mostrando hipercelularidad del cartílago (A), hiperplasia de la membrana sinovial (B-Flecha), cartílago con celularidad normal (C) y Membrana sinovial normal (D-Flecha). 
BUSTOS, L. R.; MARINO, M. I. A.; ROMERO, D. V. J.; MENDOZA, L. O. F.; LARA, A. J. \& DE LA GARZA, C. S. Análisis histopatológico del cartílago articular expuesto a metilprednisolona, ropivacaína y ácido hialurónico en un modelo animal de osteoartrosis. Int. J. Morphol., 32(4):1199-1206, 2014.

\section{DISCUSIÓN}

Una de las herramientas terapéuticas con la que cuentan los médicos ortopedistas, reumatólogos, de rehabilitación y médicos familiares capacitados para el diagnóstico y tratamiento de la osteoartrosis, es la infiltración articular. Durante el diagnóstico, el dolor limita la exploración musculo-esquelética y la introducción de una solución anestésica en la articulación permite un examen más completo que cuando el paciente presenta dolor en la región a evaluar. Y desde el punto de vista terapéutico, existen también diversos motivos para realizar infiltraciones con el objetivo de mejorar la sintomatología, prevenir, limitar o revertir la evolución natural de la enfermedad.

Los corticoesteroides han sido empleados por años en el tratamiento de la osteoartrosis y como resultado se tiene una importante experiencia clínica de su utilidad y efectividad. Estudios clínicos y meta-análisis han demostrado su eficacia basados en escalas clínicas que reflejan la mejoría sintomatológica del padecimiento desde el punto de vista del paciente (Bellamy et al., 2006). Existe poca evidencia científica, y es controversial respecto al efecto histológico y molecular de los corticoesteroides en el cartílago articular. Papacrhistou et al. (1997) en un estudio en conejos, demostraron que los corticoesteroides pueden funcionar como supresores de la síntesis de proteoglicanos, empeorando las lesiones cartilaginosas o incluso causando una lesión degenerativa en el cartílago articular sano. En nuestro estudio pudimos observar resultados similares en los que la infiltración con Acetato de Metilprednisolona tuvo un efecto negativo en el cartílago articular, contrario a nuestra hipótesis inicial, el esteroide causó lesión en el cartílago articular, elevando la puntuación respecto a la escala de Mankin. La principal diferencia fue la afección de la estructura del cartílago articular, además pudimos observar una inhibición de la síntesis de proteoglicanos coincidiendo con los resultados de Papacrhistou et al., a diferencia de Pelletier et al. (1994, 2000), quienes en diferentes estudios en los que utilizaron modelos de osteoartrosis en perros, demostraron que dosis bajas de corticoesteroides intraarticulares podían normalizar la síntesis de proteoglicanos y también podían disminuir significativamente la incidencia y severidad de la formación de osteofitos y lesiones del cartílago. Nosotros notamos un deterioro histológico del cartílago articular con una dosis única de Acetato de Metilprednisolona. En el uso de los esteroides como herramienta terapéutica en la infiltración articular debe ser tomado en cuenta que si bien ayudan en el alivio del dolor y mejoran la funcionalidad, también presentan efectos adversos histológicos en el cartílago articular.
Debido a que las dosis únicas de anestésicos locales son comúnmente administradas para alivio del dolor en el tratamiento del dolor articular, es imperativo comprender el efecto histológico de estos medicamentos en el cartílago articular, sobre la viabilidad de los condrocitos y la producción de la matriz extracelular. La condrotoxicidad de los anestésicos locales ha sido documentada en varios estudios de manera in vitro (Dragoo et al., 2008). Algunos otros estudios han reportado detrimento en la calidad del cartílago articular en modelos in vivo utilizando modelos animales (Chu et al., 2010). Nuestra hipótesis inicial con respecto al uso de anestésicos para la infiltración articular, sobre todo de Ropivacaína, fue que no tendría un efecto negativo sobre la integridad del cartílago articular. Sin embargo, observamos que la infiltración única de Ropivacaína generó alteraciones en la estructura del cartílago articular y afección en la celularidad, presentando hipercelularidad del cartílago aunada a la hiperplasia de la membrana sinovial. Estos resultados difieren con los reportados por Gomoll et al. (2006), quienes no encontraron daño en los condrocitos a largo plazo, en un periodo de 3 meses, después de infusión continua por $48 \mathrm{~h}$ de Bupivacaína $25 \%$, en un estudio experimental en modelo de artrosis en conejos. La condrolisis se define como la desaparición del cartílago articular como resultado de lisis o disolución de la matriz cartilaginosa y condrocitos y se ha reportado este efecto secundario a la infiltración articular de anestésicos locales (Anderson et al., 2010). En nuestro estudio observamos que la infiltración articular de Ropivacaína en dosis única lesiona histológicamente el cartílago articular, mas no observamos un efecto de condrolisis tal y como se define. Sin embargo, si observamos que alteraciones como la hipercelularidad, las erosiones en la superficie y la hiperplasia sinovial son más compatibles a un efecto de condrolisis y citotoxicidad que a un efecto degenerativo por osteoartrosis.

El mecanismo de acción de la viscosuplementación con Ácido Hialurónico sintético inyectado de manera intraarticular no se conoce con exactitud. Se propone que su mecanismo de acción ocurren en 2 etapas: la etapa del efecto mecánico y la etapa antiinflamatoria, anabólica y analgésica (Gosh \& Guidolin, 2002; Balazs, 1982).

Desde 1997, el Ácido Hialurónico se encuentra autorizado por la FDA para el tratamiento del dolor en la osteoartritis de rodilla, también ha sido aprobado por el Colegio Americano de Reumatología y por la Liga Europea contra el reumatismo como opción terapéutica para el alivio del dolor en la osteoartrosis (Zhang et al., 2005; American College of Rheumatology, 2000). 
La bibliografía publicada de medicina basada en evidencia respecto al empleo de ácido Hialurónico no es concluyente en cuanto a condroprotección o reversión de la osteoartrosis. Numerosos estudios han reportado un beneficio clínico sostenido en la rodi1la al administrar ácido Hialurónico, mientras que otras investigaciones no lograron demostrar una diferencia significativa al comparar a este polisacárido con solución salina y placebo, cuestionando la verdadera magnitud del efecto terapéutico del ácido Hialurónico, de tal manera que se llevaron a cabo varios meta-análisis que pretendían esclarecer dicha cuestión (Arrich et al., 2005). Sin embargo, los resultados han sido muy variables y basados principalmente al efecto clínico y pocos estudios se han realizado para valorar el efecto histológico, sin llegar a una conclusión convincente.
En nuestro estudio observamos que en el grupo de Ácido Hialurónico, el cartílago articular presentó menos deterioro histológico respecto al grupo control, principalmente en la integridad del cartílago articular, la regularidad de su superficie y la producción de proteoglicanos por los condrocitos. Estos resultados son similares a los reportados por Barbucci et al. (2002), quienes también encontraron mejoría en el contenido de condrocitos y morfología de la matriz cartilaginosa en rodillas con osteoartrosis en las que se utilizó ácido Hialurónico.

El Ácido Hialurónico es capaz de aliviar la sintomatología y mejorar la calidad de vida del paciente según el grado de lesión que esté presente cartílago articular. Nuestros resultados sugieren que la administración del ácido Hialurónico, podría retrasar el proceso de una osteoartrosis leve.

BUSTOS, L. R.; MARINO, M. I. A.; ROMERO, D. V. J.; MENDOZA, L. O. F.; LARA, A. J. \& DE LA GARZA, C. S. Histopathological analysis of the articular cartilage exposed to methylprednisolone, ropivacaine and hyaluronic acid in an animal model of osteoarthrosis. Int. J. Morphol., 32(4):1199-1206, 2014.

SUMMARY: Osteoarthritis is a musculoskeletal condition with a high prevalence, increasing with the aging of population. The intraarticular infiltration of substances to relieve the symptoms of osteoarthritis is a common practice in medical practice. Although symptoms improved with the infiltration of local anesthetics, corticosteroids and supplements, it is still uncertain what effect these substances have on the integrity of articular cartilage. This study explores the macroscopic and histological effects of infiltration of Ropivacaine, Methylprednisolone and Hyaluronic Acid on articular cartilage in a model of chemical osteoarthritis in rabbits ( $\mathrm{n}=24$ ). Our results indicate that in the infiltrated groups with Methylprednisolone $(n=8)$ and Ropivacaine $(n=8)$ the structure of articular cartilage present more severe alterations with respect to its control group and an important decrease in the synthesis of extracellular matrix. Ingroup infiltrated with hyaluronic acid $(n=8)$, macroscopic and histological changes of articular cartilage improved with respect to its control group, presenting a normal structure and normal extracellular matrix synthesis.

\section{KEY WORDS: Intraarticular Infiltration; Articular Cartilage; Osteoarthritis.}

\section{REFERENCIAS BIBLIOGRÁFICAS}

American College of Rheumatology. Recommendations for the medical management of osteoarthritis of the hip and knee: 2000 update. American College of Rheumatology Subcommittee on Osteoarthritis Guidelines. Arthritis Rheum., 43(9):1905-15, 2000 .

Anderson, S. L.; Buchko, J. Z.; Taillon, M. R. \& Ernst, M. A. Chondrolysis of the glenohumeral joint after infusion of bupivacaine through an intra-articular pain pump catheter: a report of 18 cases. Arthroscopy, 26(4):451-61, 2010.

Arrich, J.; Piribauer, F.; Mad, P.; Schimd, D.; Klaushofer, K. \& Müllner, M. Intra-articular hyaluronic acid for the treatment of osteoarthritis of the knee: systematic review and metaanalysis. CMAJ, 172(8):1039-43, 2005.

Balazs, E. The physical properties of synovial fluid and the specific role of hyaluronic acid. In: Helfet, A. J. (Ed.). Disorders of the Knee. 2nd ed. Philadelphia, J. B. Lippincott, 1982. pp.61-74.
Barbucci, R.; Lamponi, S.; Borzacchiello, A.; Ambrosio, L.; Fini, M.; Torricelli, P. \& Giardino, R. Hyaluronic acid hydrogel in the treatment of osteoarthritis. Biomaterials, 23(23):4503-13, 2002.

Bellamy, N.; Campbell, J.; Robinson, V.; Gee, T.; Bourne, R. \& Wells, G. Intraarticular corticosteroid for treatment of osteoarthritis of the knee. Cochrane Database Syst. Rev., (2):CD005328, 2006

Brandt, K.; Lohmander, L. \& Doherty, M. The concept of osteoarthritis as failure of the diarthrodial joint. In: Brandt, K.; Doherty, M. \& Lohmander, L. (Eds.). Osteoarthritis. Oxford, Oxford University Press, 1998. pp.70-4.

Chu, C. R.; Coyle, C. H.; Chu, C. T.; Szczodry, M.; Seshadri, V.; Karpie, J. C.; Cieslak, K. M. \& Pringle, E. K. In vivo effects of single intra-articular injection of $0.5 \%$ bupivacaine on articular cartilage. J. Bone Joint Surg. Am., 92(3):599-608, 2010. 
BUSTOS, L. R.; MARINO, M. I. A.; ROMERO, D. V. J.; MENDOZA, L. O. F.; LARA, A. J. \& DE LA GARZA, C. S. Análisis histopatológico del cartílago articular expuesto a metilprednisolona, ropivacaína y ácido hialurónico en un modelo animal de osteoartrosis. Int. J. Morphol., 32(4):1199-1206, 2014.

Dragoo, J. L.; Korotkova, T.; Kanwar, R. \& Wood, B. The effect of local anesthetics administered via pain pump on chondrocyte viability. Am. J. Sports Med., 36(8):1484-8, 2008.

Elders, M. J. The increasing impact of arthritis on public health. $J$. Rheumatol. Suppl., 60:6-8, 2000.

Fahmy, N. R.; Williams, E. A. \& Noble, J. Meniscal pathology and osteoarthritis of the knee. J. Bone Joint Surg., 65(1):24-8, 1983.

Felson, D. T.; Lawrence, R. C.; Dieppe, P. A.; Hirsch, R.; Helmick, C. G.; Jordan, J. M.; Kington, R. S.; Lane, N. E.; Nevitt, M. C.; Zhang, Y.; Sowers, M.; McAlindon, T.; Spector, T. D.; Poole, A. R.; Yanovski, S. Z.; Ateshian, G.; Sharma, L.; Buckwalter, J. A.; Brandt, K. D. \& Fries, J. F. Osteoarthritis: new insights. Part 1: the disease and its risk factors. Ann. Intern. Med., 133(8):635-45, 2000.

Gomoll, A. H.; Kang, R. W.; Williams, J. M.; Bach, B. R. \& Cole, B. J. Chondrolysis after continuous intra-articular bupivacaine infusion: An experimental model investigating chondrotoxicity in the rabbit shoulder. Arthroscopy, 22(8):813-9, 2006.

Gosh, P. \& Guidolin, D. Potential mechanism of action of intraarticular hyaluronan therapy in osteoarthritis: are the effects molecular weight dependent? Semin. Arthritis Rheum., 32(1):10-37, 2002.

Kikuchi, T.; Sakuta, T. \& Yamaguchi, T. Intra-articular injection of collagenase induces experimental osteoarthritis in mature rabbits. Osteoarthritis Cartilage, 6(3):177-86, 1998.

Kuettner, K. E. \& Goldberg, V. M. Osteoarthritic disorders. Rosemont, American Academy of Orthopaedic Surgeons, 1995. pp.21-5.

Meachim, G. Articular cartilage lesions in osteo-arthritis of the femoral head. J. Pathol., 107(3):199-210, 1972.

Mankin, H. J.; Dorfman, H.; Lippiello, L. \& Zarins, A. Biochemical and metabolic abnormalities in articular cartilage from osteoarthritic human hips. II. Correlation of morphology with biochemical and metabolic data. J. Bone Joint Surg. Am., 53(3):523-37, 1971

Papacrhistou, G.; Anagnostou, S. \& Katsorhis, T. The effect of intraarticular hydrocortisone injection on the articular cartilage of rabbits. Acta Orthop. Scand. Suppl., 275:132-4, 1997.

Pelletier, J. P.; Lajeunesse, D.; Jovanovic, D. V.; Lascau-Coman, V.; Jolicoeur, F. C.; Hilal, G.; Fernandes, J. C. \& MartelPelletier, J. Carprofen simultaneously reduces progression of morphological changes in cartilage and subchondral bone in experimental dog osteoarthritis. J. Rheumatol., 27(12):2893902, 2000.

Pelletier, J. P.; Mineau, F.; Raynauld, J. P.; Woessner, J. F. Jr.; GunjaSmith, Z. \& Martel-Pelletier, J. Intraarticular injections with methylprednisolone acetate reduce osteoarthritic lesions in parallel with chondrocyte stromelysin synthesis in experimental osteoarthritis. Arthritis Rheum., 37(3):414-23, 1994.

Piper, S. L. \& Kim, H. T. Comparison of ropivacaine and bupivacaine toxicity in human articular chondrocytes. J. Bone Joint Surg. Am., 90(5):986-91, 2008.

Poole, C. A.; Matsouka, A. \& Schofield, J. R. Chondrons from articular cartilage. III. Morphologic changes in the cellular microenvironment of chondrons isolated from osteoarthritic cartilage. Arthritis Rheum., 34(1):22-35, 1991.

Saito, I.; Koshino, T.; Nakashima, K.; Uesugi, M. \& Saito, T. Increased cellular infiltrate in inflammatory synovia of osteoarthritic knees. Osteoarthritis Cartilage, 10(2):156-62, 2002.

Yoshioka, M.; Coutts, R. D.; Amiel, D. \& Hacker, S. A. Characterization of a model of osteoarthritis in the rabbit knee. Osteoarthritis Cartilage, 4(2):87-98, 1996.

Zhang, W.; Doherty, M.; Arden, N.; Bannwarth, B.; Bijlsma, J.; Gunther, K. P.; Hauselmann, H. J.; Herrero-Beaumont, G.; Jordan, K.; Kaklamanis, P.; Leeb, B.; Lequesne, M.; Lohmander, S.; Mazieres, B.; Martin-Mola, E.; Pavelka, K.; Pendleton, A.; Punzi, L.; Swoboda, B.; Varatojo, R.; Verbruggen, G.; Zimmermann-Gorska, I.; Dougados, M. \& EULAR Standing Committee for International Clinical Studies Including Therapeutics (ESCISIT). EULAR evidence based recommendations for the management of hip osteoarthritis: report of a task force of the EULAR Standing Committee for International Clinical Studies Including Therapeutics (ESCISIT). Ann. Rheum. Dis., 64(5):669-81, 2005.

\author{
Dirección para Correspondencia: \\ Santiago De La Garza Castro \\ Campus de la Salud, \\ Ave. Gonzalitos, Col. \\ Mitras Centro \\ Monterrey, Nuevo León \\ C.P. 66460 \\ MÉXICO
}

Email: doc-santiago@hotmail.com

Recibido : 21-02-2014

Aceptado: 25-07-2014 